\title{
Sensitivity and specificity of ECG criteria of left atrial enlargement in comparison to cardiac magnetic resonance in patients with hypertrophic cardiomyopathy
}

Mariusz Kłopotowski ${ }^{1, A-F}$, Anna Jasińska ${ }^{2, A-D}$, Ewelina Jarmus ${ }^{3, A-D}$, Mateusz Śpiewak ${ }^{4, B-C}$, Jacek Jamiołkowski5 ${ }^{5, C}$, Aleksandra Kwapiszewska, ${ }^{1, \mathrm{~B}-\mathrm{C}}$, Rafał Baranowski ${ }^{6, \mathrm{E}-\mathrm{F}}$, Łukasz A. Małek ${ }^{7, \mathrm{C}-\mathrm{F}}$

A - Research concept and design, B - Collection and/or assembly of data, C - Data analysis and interpretation, D - Writing the article, E - Critical revision of the article, F - Final approval of article

1 Department of Interventional Cardiology and Angiology, Institute of Cardiology, Warsaw, Poland

2 Department of Acquired Cardiac Defects, Institute of Cardiology, Warsaw, Poland.

3 Mazowiecki Szpital Specjalistyczny, Radom, Poland

4 Department of Coronary Heart Disease and Structural Heart Diseases, Institute of Cardiology, Warsaw, Poland

5 Magnetic Resonance Unit, Institute of Cardiology, Warsaw, Poland

6 Public Health Department, Medical University of Bialystok, Bialystok, Poland.

7 Department of Cardiac Arrhythmia, Institute of Cardiology, Warsaw, Poland

8 Collegium Masoviense, Żyrardów, Poland

Address for correspondence:

Mariusz Kłopotowski, MD PhD, Department of Interventional Cardiology and Angiology, Institute of Cardiology, Alpejska str 42, 04-628, Warsaw, Poland, e-mail: mklopotowski@hotmail.com, tel: +48-22-34-34- 267, fax: +48-22- 613-38- 19

\section{Structured abstract}

Background. In patients with hypertrophic cardiomyopathy (HCM), left atrial (LA) enlargement has been related to increased risk of sudden cardiac death. Cardiac magnetic resonance (CMR) is the method of choice in the assessment of LA size and volume. However, initial assessment of LA enlargement is often made by means of ECG criteria including P wave analysis in leads II and V1. The aim of the study was to assess the sensitivity and specificity of ECG criteria for LA enlargement in relation to CMR.

Materials and methods: The study included 236 patients (62.7\% male, median age 47 years) with documented HCM and sinus rhythm on 12-lead ECG who underwent CMR study. ECGs were digitally analyzed for the following criteria: $P$ wave duration in lead II $>120 \mathrm{~ms}$ or area of negative P-terminal force in $\mathrm{V} 1 \geq 40 \mathrm{~ms} . \mathrm{mm}$. LA volume index (LAVI) $>53 \mathrm{ml} / \mathrm{m} 2$ was used as a cut-off value of LA enlargement in CMR studies.

\section{Introduction}

Hypertrophic cardiomyopathy (HCM) is a genetically determined heart disease characterized by mostly asymmetric hypertrophy of the left ventricle muscle. The disease may have a highly variable clinical course ranging from lack of clinical symptoms and life expectancy similar to the healthy population to end-stage heart failure and/or sudden cardiac death (SCD). Paramount factors in management of patients with HCM include assessment of the risk of SCD and patient stratification towards preventive ICD placement ${ }^{[1,2]}$. Recently a HCM-risk model used to stratify patients has been proposed and endorsed by the European Society of Cardiology (ESC).
Results: LA enlargement on ECG was found in 109 patients $(46.2 \%)$ and on CMR in 48 patients (20.3\%). Sensitivity, specificity, and positive and negative predictive values of LA enlargement based on P wave characteristics in lead II were $54.1 \%, 67.4 \%, 36.7 \%$ and $80.8 \%$ and in lead V1 $32.8 \%$, $80.0 \%, 36.4 \%$ and $77.3 \%$.

Conclusions: Current ECG criteria of LA enlargement have limited diagnostic value in detection of LA enlargement due to their low sensitivity and only moderate specificity.

\section{Key words}

genetic heart disorder, non-invasive test, assessment, imaging

It is based on several clinical and imaging parameters including left atrial (LA) size ${ }^{[3]}$. This is due to the fact that in several studies it has been shown that LA enlargement increases the risk of atrial fibrillation and $\mathrm{SCD}^{[4]}$. Patients with a large LA volume and those with a sudden increase of LA volume are at particularly high risk of cerebrovascular events ${ }^{[4]}$.

Although the most commonly used method of LA size assessment included in the HCM-risk model is transthoracic echocardiography, initial assessment may be based on electrocardiographic parameters. On the other hand, cardiac magnetic resonance (CMR) is considered as the method of choice in non-invasive assessment of LA size and volume ${ }^{[5,6]}$. 
Moreover, in recent years this method has been consistently demonstrated to provide incremental diagnostic and prognostic information in a HCM setting ${ }^{[6]}$.

Therefore, the aim of the study was to assess the sensitivity and specificity of ECG criteria for LA enlargement in relation to $\mathrm{CMR}$.

\section{Materials and methods}

\section{Study group}

The study group included 236 patients with documented $\operatorname{HCM}$ (62.7\% male, median age 47 years) who underwent CMR evaluation using a Siemens 1.5T Avanto scanner for further SCD risk stratification between 2008 and 2014. The diagnosis of $\mathrm{HCM}$ was based on the presence of asymmetric left ventricular hypertrophy (>15 mm) on transthoracic echocardiography after exclusion of secondary causes of hypertrophy ${ }^{[2]}$. Baseline characteristics of the studied population were collected.

\section{ECG acquisition and analysis}

For the purpose of this study routine periodic ECG tracings performed in the earliest period after CMR evaluation were chosen. They were assessed using the Sentinel software (Spacelabs Healthcare, Hertford, UK) allowing for detailed measurement of $\mathrm{P}$ wave duration and amplitude in leads II and V1. Patients with atrial fibrillation on ECG were excluded from the study. Electrocardiographic criteria of LA enlargement were: duration of the P wave in lead II $>120 \mathrm{ms,}$ and area of negative P-terminal force in V1 $>40 \mathrm{~ms} . \mathrm{mm}$, as reported previously and recommended by the Working Group of the Polish Cardiac Society (Figure 1) ${ }^{[7,8]}$.

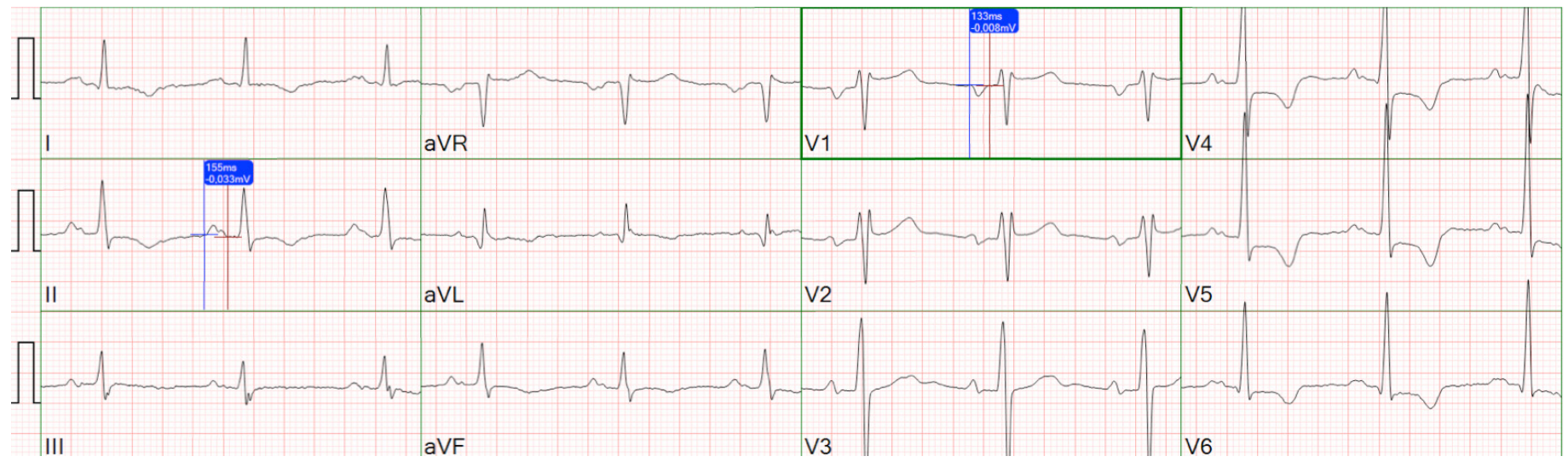

Figure 1. Examples of ECG analysis for determination of the presence of LA enlargement criteria: duration of the $\mathrm{P}$ wave in lead II \&gt; $120 \mathrm{~ms}$ or area of negative $P$ wave force in $\mathrm{V} 1 \geqslant 40 \mathrm{~ms} . \mathrm{mm}$

\section{CMR acquisition and analysis}

CMR assessment was performed by two experienced readers with at least 8 years of expertise in the field [L.A.M, M.S.] holding a Level 3 certificate of competence in CMR issued by the Working Group of the ESC. Dedicated software was used for that purpose (MASS v7.6, Medis, Leiden, the Netherlands). Left atrial measurements were made on 2-chamber, 3-chamber and 4-chamber gated breath-hold steady state free precession (SSFP) images during the ventricular end-systolic phase. For 2-chamber and 4-chamber views two orthogonal diameters of the LA as well as its area obtained by manual delineation of the LA contours along the mitral valve annulus and atrial walls were calculated (Figure 2). The 3-chamber measurements included LA area delineation and antero-posterior LA diameter. These values were used for calculation of the LA volume according to the equation proposed by Maceira et al..$^{[9]}$, which correlated best with LA volume obtained from 3-dimensional measurements. Left atrial volume normalized to body surface area, called left atrial volume index (LAVI), of $53 \mathrm{ml} / \mathrm{m} 2$ represented the upper limit of normality in that study and therefore was used to define LA enlargement in our study.

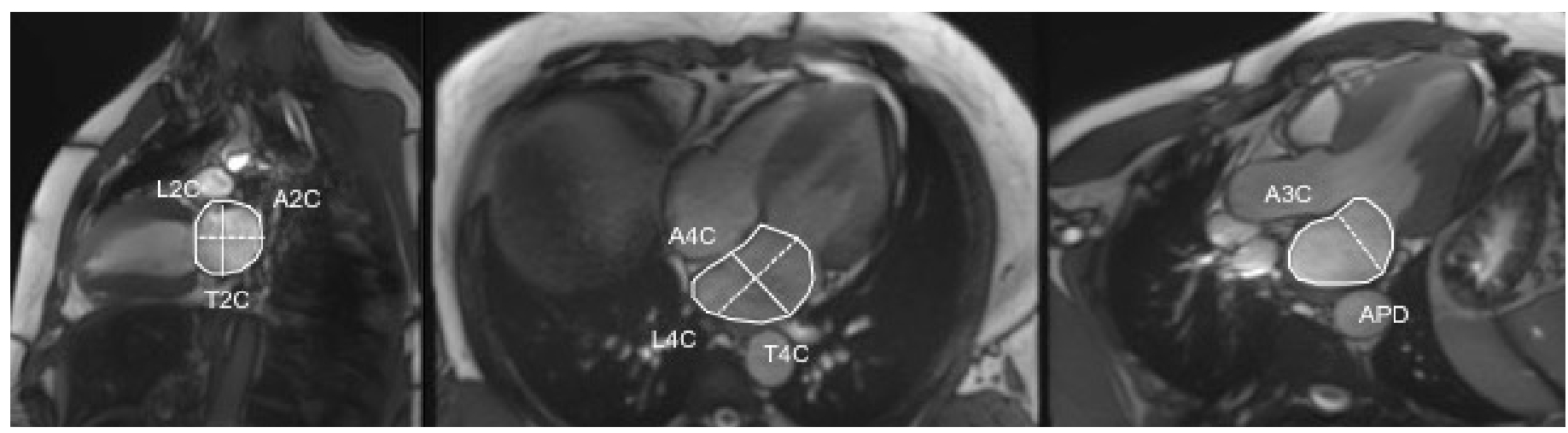

Figure 2. CMR values used for calculation of LA volume according to an equation proposed elsewhere: LAV = 3.31+[1.9*A3]+[1.1*A4]+[1.1*TD2]+[0.9*TD4]-[1.7*APD], where LAV is LA volume $(\mathrm{mL}), A 3$ is area in the three-chamber view, $A 4$ is area in the four-chamber view (both in $\mathrm{cm}^{2}$ ), TD2 is transverse diameter in the two-chamber view, TD4 is transverse diameter in the four-chamber view and APD is antero-posterior diameter (all in $\mathrm{cm}$ ). 


\section{Statistical analysis}

All results for categorical variables are presented as number and percentage and for continuous variables as median and interquartile range (IQR). Spearman's test was applied to assess correlation. Receiver operating characteristic (ROC) curves were used to compare the ability of ECG criteria of LA enlargement to predict increased LA volume defined using CMR. Sensitivity, specificity, and positive and negative predictive values were calculated. Statistical analyses were performed with IBM SPSS Statistics package (IBM Corp. Released 2011. IBM SPSS Statistics for Windows, Version 20.0. Armonk, NY: IBM Corp.). P-values smaller than 0.05 were considered statistically significant. All provided p-values relate to 2 -tailed tests.

\section{Results}

Baseline characteristics of the studied population are presented in Table 1. Electrocardiographic criteria for LA

Table 1. Baseline characteristics of studied population
Studied population
$n=236$

enlargement were met by 109 patients (46.2\%) and for LA enlargement on CMR by 48 patients $(20.3 \%)$. When only a single lead ECG criterion was taken into account, LA enlargement was observed in 90 patients $(38.2 \%)$ based on $\mathrm{P}$ wave characteristics in lead II and in 55 patients $(23.3 \%)$ based on $\mathrm{P}$ wave characteristics in lead V1.

Positive but weak correlations were found between LAVI and ECG criteria. We also assessed the correlation between LAVI and more often clinically used 2-dimensional CMR measures of LA size. The highest correlations were found for 3-chamber and 4-chamber LA areas, with a lower correlation for 2-chamber LA area (Table 2).

Table 2. Correlation between LAVI and ECG criteria of LA enlargement as well as selected 2-dimensional CMR parameters of LA size

\section{Correlation
coefficient $(r)$}

ECG criteria

\begin{tabular}{lll}
\hline P wave $>120$ ms in lead II & 0.31 & $<0.0001$ \\
$\begin{array}{l}\text { Area of negative } P \text { wave } \\
\text { force in } \mathrm{V} 1 \geqslant 40 \text { ms.mm }\end{array}$ & 0.34 & $<0.0001$ \\
Selected CMR parameters* & & \\
\hline
\end{tabular}

$\begin{array}{lcc}\text { A4 } & 0.79 & <0.0001 \\ \text { A3 } & 0.83 & <0.0001 \\ \text { A2 } & 0.67 & <0.0001 \\ \text { *A2, A3 and A4 are LA areas in the 2-, 3- and 4-chamber view respectively }\end{array}$

Receiver operating curves for ECG criteria of LA enlargement in prediction of LAVI $>53 \mathrm{ml} / \mathrm{m} 2$ are presented in Figure 3. There was no significant difference between the two areas under the curve $(p=0.47)$. Sensitivity, specificity, and positive and negative predictive value of LA enlargement based on P wave duration in lead II were $54.1 \%, 67.4 \%, 36.7 \%$ and $80.8 \%$ and based on duration and amplitude of the P wave in lead V1 were $32.8 \%, 80.0 \%, 36.4 \%$ and $77.3 \%$.

\section{Discussion}

We have shown in patients with hypertrophic cardiomyopathy that electrocardiographic criteria for LA enlargement detection have only limited diagnostic value. This is mainly due to low sensitivity of these criteria and therefore a low positive predictive value, signifying that ECG cannot be used as a screening method for LA enlargement detection in this group 
of patients. Moreover, contrary to other reports discussed below, if the ECG criteria are met, they have only moderate specificity. We have also demonstrated that there is a significant difference in diagnostic accuracy between the two ECG criteria, favoring one criterion over another.

Figure 3. Receiver operating curves for ECG criteria in discrimination of $\mathrm{LAVl}>53 \mathrm{ml} / \mathrm{m}^{2}$

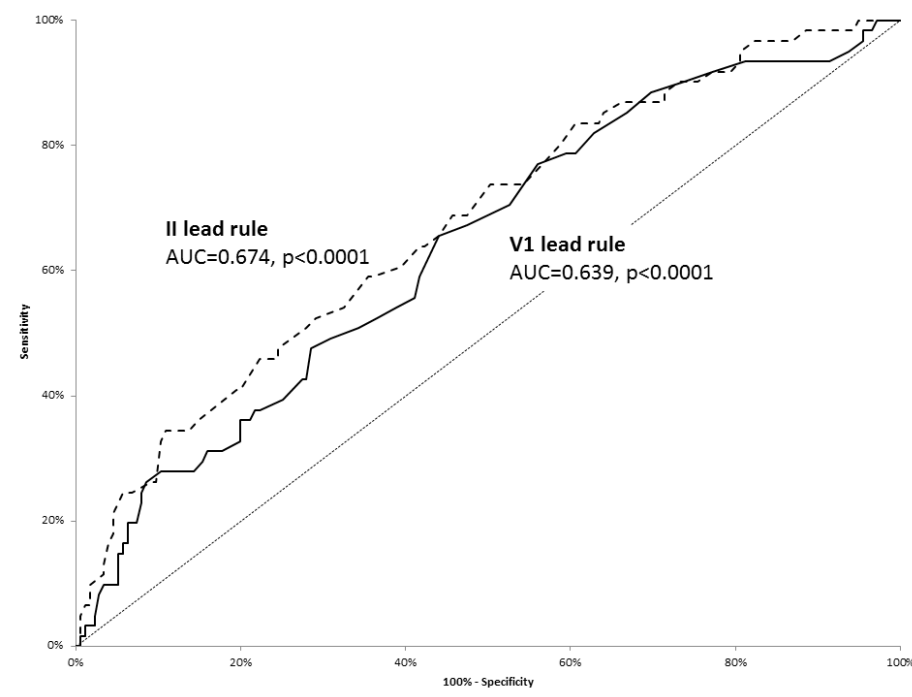

In the past, several studies have assessed the accuracy of ECG criteria in prediction of LA enlargement using echocardiography and often demonstrated conflicting results ${ }^{[10-13]}$. Most of them reported relatively low sensitivity but much higher specificity of these criteria, often approximating $100 \%{ }^{[10,12]}$.

Introduction of CMR into clinical practice verified previous electrocardiographic and echocardiographic estimates of atrial size based on 2-dimensional measurements by application of 3-dimensional, volumetric atrial size quantification. Previous studies comparing electrocardiographic criteria with CMR for LA size assessment in other patient populations produced results similar to ours. In the majority of these studies the prevalence of LA enlargement by CMR was much lower than in ECG and varied between 20 and $30 \%{ }^{[14-16]}$. It was higher only in the study by Burekaam et al., but the authors decided to choose a lower cut-off value for LA enlargement as compared to our and other studies ${ }^{[16]}$. Tsao et al. analyzed 275 patients referred for CMR between 2001 and 2004 for various clinical indications (mainly left ventricular function assessment and pulmonary vein assessment) and found low sensitivity but high specificity of the lead V1 criterion (35\% and $88 \%$ respectively) as well as surprisingly higher sensitivity than specificity for the lead II criterion ( $84 \%$ vs. $35 \%)^{[14]}$. Rodrigues et al. also demonstrated higher specificity than sensitivity of ECG criteria, but the differences between sensitivity and specificity were larger than in our study. Contrary to our study, the authors observed higher specificity for the lead II than the lead V1 criterion $[91 \% \text { vs. } 81 \%]^{[15]}$. They also observed that obesity was a strong attenuation factor for specificity. Similarly, in the study by Bureekam et al., sensitivity of individual ECG criteria for LA enlargement was low: $49.6 \%$ for $P$ wave $>120$ $\mathrm{ms}$ in lead II and $30.8 \%$ for biphasic $\mathrm{P}$ wave in $\mathrm{V} 1^{[16]}$. However, specificity of individual ECG criteria for LA enlargement in that study was $100 \%$.

In summary, contrary to other echocardiographic and CMR studies, our results questioned the high specificity of ECG criteria, suggesting their overall low diagnostic accuracy.

Our study has some limitations. Due to the retrospective nature of our analysis we were not able to use a truly volumetric assessment of LA size as a comparator. However, the results were based on an LA volume estimate based on the previously validated equation ${ }^{[9]}$. Secondly, we analyzed only two ECG criteria of LA enlargement, but we wanted to limit our analysis to current recommendations of the Polish Society of Cardiology. ${ }^{[8]}$

\section{Conclusions}

Assessment of LA size is of paramount importance in patients with $\mathrm{HCM}$ due to its influence on SCD risk stratification. Although there is a positive correlation between LA volume in CMR and features of LA enlargement on ECG, current ECG criteria have only limited diagnostic value due to low sensitivity and only moderate specificity.

\section{REFERENCES}

1. Maron BJ, Casey SA, Hauser RG, Aeppli DM. Clinical course of hypertrophic cardiomyopathy with survival to advanced age. J Am Coll Cardiol 2003;42:882-8.

2. Elliott PM, Anastasakis A, Borger MA, Borggrefe M, Cecchi F, Charron P, et al. 2014 ESC Guidelines on diagnosis and management of hypertrophic cardiomyopathy: the Task Force for the Diagnosis and Management of Hypertrophic Cardiomyopathy of the European Society of Cardiology (ESC). Eur Heart J 2014;35(39):2733-79.

3. O'Mahony C, Jichi F, Pavlou M, Monserrat L, Anastasakis A, Rapezzi C, et al. A novel clinical risk prediction model for sudden cardiac death in hypertrophic cardiomyopathy (HCM Risk-SCD). Eur Heart J 2014; 35: 2010-2020.

4. Losi MA, Betocchi S, Barbati G, Parisi V, Tocchetti CG, Pastore F, et al. Prognostic significance of left atrial volume dilatation in patients with hypertrophic cardiomyopathy. J Am Soc Echocardiogr 2009;22:76-81.

5. Panovsky R, Pleva M, Feitova V, Kruzliak P, Meluzin J, Kincl V, et al. Left atrium assessment: the evolving role of MRI. J Cardiovasc Med (Hagerstown). 2015;16:671-80.

6. Maron MS. Clinical utility of cardiovascular magnetic resonance in hypertrophic cardiomyopathy. J Cardiovasc Magn Reson 2012;14:13. 
7. Alpert MA, Munuswamy K. Electrocardiographic diagnosis of left atrial enlargement. Arch Intern Med 1989;149:1161-5.

8. Baranowski R, Wojciechowski D, Kozłowski D, Kukla P, Kurpesa M, Lelakowski J, et al. Electrocardiographic criteria for diagnosis of the heart chamber enlargement, necrosis and repolarisation abnormalities including acute coronary syndromes. Experts\&\#39; group statement of the Working Group on Noninvasive Electrocardiology and Telemedicine of Polish Society of Cardiology. Kardiol Pol 2016;74(8):812-9.

9. Maceira A, Cosín-Sales J, Roughton M, Prasad SK, Pennell DJ. Reference left atrial dimensions and volumes by steady state free precession cardiovascular magnetic resonance. J Cardiovasc Magn Reson 2010;12:65.

10. Ikram H, Drysdale P, Bones PJ, Chan W: The non-invasive recognition of left atrial enlargement: comparison of electro- and echocardiographic measurements. Postgrad Med J 1977;53(621):356-9.

11. Waggoner AD, Adyanthaya AV, Quinones MA, Alexander JK. Left atrial enlargement. Echocardiographic assessment of electrocardiographic criteria. Circulation 1976;54(4):553-7.
12. Munuswamy K, Alpert MA, Martin RH, Whiting RB, Mechlin NJ. Sensitivity and specificity of commonly used electrocardiographic criteria for left atrial enlargement determined by M-mode echocardiography. Am J Cardiol 1984;53(6):829-32.

13. Hazen MS, Marwick TH, Underwood DA. Diagnostic accuracy of the resting electrocardiogram in detection and estimation of left atrial enlargement: an echocardiographic correlation in 551 patients. Am Heart J 1991;122:823-8.

14. Tsao CW, Josephson ME, Hauser TH, O'Halloran TD, Agarwal A,Manning WJ, et al. Accuracy of electrocardiographic criteria for atrial enlargement: validation with cardiovascular magnetic resonance. J Cardiovasc Magn Reson 2008;10:7.

15. Rodrigues JC, Erdei T, Dastidar AG, McIntyre B, Burchell AE, Ratcliffe LE, et al. Electrocardiographic detection of hypertensive left atrial enlargement in the presence of obesity: re-calibration against cardiac magnetic resonance. J Hum Hypertens. 2016 [Epub ahead of print]

16. Bureekam S, Boonyasirinant T. Accuracy of left atrial enlargement diagnosed by electrocardiographyas compared to cardiac magnetic resonance in hypertensive patients. J Med Assoc Thai 2014;97 Suppl 3:S132-8. 